\title{
Estudo normativo e avaliação da influência da idade no potencial evocado auditivo em cães sem raça definida
}

\author{
[Normative study and evaluation of age influence on Brainstem auditory \\ evoked potentials in mixed breed dogs] \\ M.I.P. Palumbo ${ }^{1}$, L.A.L. Resende ${ }^{2}$, A.S. Borges $^{3} *$ \\ ${ }^{\mathbf{1}}$ Aluna de pós-graduação - Faculdade de Medicina Veterinária e Zootecnia - FMVZ-Unesp - Botucatu, SP \\ ${ }^{2}$ Faculdade de Medicina de Botucatu - FMB-Unesp - Botucatu, SP \\ ${ }^{3}$ Faculdade de Medicina Veterinária e Zootecnia - FMVZ-Unesp - Botucatu, SP
}

\begin{abstract}
RESUMO
O objetivo deste trabalho foi realizar estudo normativo dos potenciais evocados auditivos obtidos de 34 cães sem raça definida e avaliar a influência da idade nos resultados obtidos. Os animais foram divididos em dois grupos de diferentes faixas etárias e o potencial evocado auditivo foi realizado com um estímulo de $85 \mathrm{~dB}$. O grupo 1 incluiu 16 cães com idades entre um e oito anos e o grupo 2, 18 animais com idades acima de oito anos. O comprimento e o diâmetro da cabeça foram mensurados e não houve diferença estatística entre os dois grupos. No grupo 1, as médias das latências das ondas I, III e V foram: 1,13; 2,64 e 3,45ms; e dos intervalos I-III, III-V e I-V foram 1,51; 0,81 e 2,32ms, respectivamente. No grupo 2, as médias das latências das ondas I, III e V foram 1,15; 2,62 e 3,55ms; e dos intervalos I-III, III-V e I-V foram 1,47; 0,93 e 2,40ms, respectivamente. As latências observadas neste estudo foram semelhantes a estudos prévios realizados por outros autores. Nos grupos etários analisados e nas condições preestabelecidas deste estudo, a idade influenciou a latência da onda $\mathrm{V}$ e, consequentemente, modificou os intervalos III-V e I-V, portanto deve-se considerar essa variante na interpretação dos resultados do BAEP em cães.
\end{abstract}

Palavras- chave: potencial evocado auditivo, idade, cães

\begin{abstract}
The aim of this paper was to obtain normative data of auditory evoked potentials from 34 mixed breed dogs and evaluate the age influence. The animals were divided in two groups of different ages and auditory evoked potential was performed with a $85 \mathrm{~dB}$ stimulus intensity. Group 1 included 16 dogs between 1 and 8 years of age, and group 2 included 18 dogs with over 8 years of age. The length and head diameter were measured and there was no statistical difference between the two groups. In group 1, mean latencies of waves I, III, and V were 1.13; 2.64, and 3.45ms, and the intervals I-III, III-V, and I-V were 1.51; 0.81, and $2.32 \mathrm{~ms}$, respectively. In group 2, the mean latencies of waves I, III and V were 1.15, 2.62, and 3.55ms, and the intervals I-III, III-V, and I-V were 1.47, 0.93, and 2.40ms, respectively. The latencies observed in this study were similar to previous studies conducted by other authors. It was observed that significant differences were present for wave $V$ and intervals III-V and I-V latencies when comparing groups with different ages, consequently this characteristic must be considered during BAEP result interpretation.
\end{abstract}

Keywords: auditory evoked potentials, hearing, age, dogs

Recebido em 3 de março de 2012

Aceito em 6 de março de 2013

* Autor para correspondência (corresponding author)

E-mail: asborges@fmvz.unesp.br 


\section{INTRODUÇÃO}

O potencial evocado auditivo de tronco encefálico (BAEP - Brainstem Auditory Evoked Potential) representa atividade elétrica gerada no oitavo nervo craniano e de projeções centrais das vias auditivas, com a utilização de eletrodos que detectam pequenas alterações na atividade bioelétrica em resposta a estímulo acústico específico, frequentemente apresentado na forma de um "clique" (Marshall, 1986; Uzuka et al., 1996; Shiu et al., 1997; Wilson e Mills, 2005).

O resultado do BAEP de um cão normal consiste em quatro a sete ondas nomeadas por algarismos romanos, que aparecem nos primeiros $10 \mathrm{~ms}$ após o estímulo, e cada uma delas, provavelmente, representa um local específico da via auditiva (Luttgen, 1994; Shiu et al., 1997; Webb, 2009). Apesar de existir discordância entre os autores sobre o local de geração das ondas registradas nos exames, trabalhos recentes sugerem que as ondas I a III são geradas principalmente pelo oitavo nervo craniano, núcleo coclear e complexo olivar superior; enquanto as ondas IV a VII são geradas, preferencialmente, por uma ou mais das seguintes estruturas: lemnisco lateral, colículo caudal ou núcleo geniculado medial (Wilson e Mills, 2005; Webb, 2009).

Durante as últimas décadas, o BAEP tem sido amplamente utilizado com diferentes objetivos em instituições veterinárias de outros países. Mais comumente, o BAEP é usado para auxiliar o diagnóstico de diversas formas de surdez, particularmente a hereditária e a senil (Strain et al., 1992; Luttgen, 1994; Strain, 1996), para determinar o limiar acústico de animais (Kay et al., 1984; Marshall, 1986) e em estudos para avaliar a ototoxicidade de alguns medicamentos (Uzuka et al., 1996). O exame também auxilia a avaliação da integridade do tronco encefálico, podendo ser usado em animais com trauma craniano, déficits de equilíbrio e em outras condições em que o animal está comatoso e os reflexos de nervos cranianos não podem ser avaliados (Luttgen, 1994; Steiss et al., 1994).

Estudos realizados em cães indicam que a idade pode influenciar os registros de potenciais evocados auditivos (Knowles et al., 1988; Knowles et al., 1989; Shimada et al., 1998; Haar et al., 2008). Apesar de os dados observados em diferentes publicações sobre o uso do BAEP em medicina veterinária serem relativamente uniformes, é necessário cada laboratório realizar um estudo de padronização da técnica, de acordo com o equipamento utilizado. Desta maneira, o objetivo do presente estudo foi normatizar o uso do BAEP em cães sem raça definida e avaliar a influência da idade nos potenciais obtidos.

\section{MATERIAL E MÉTODOS}

Após aprovação pelo comitê de ética da Unesp, Botucatu (protocolo 117/2009), foram selecionados 34 cães sem raça definida, sendo 16 com idades entre um e oito anos (Grupo 1) e 18 com idades acima de oito anos (Grupo 2), provindos de proprietários que forneceram consentimento prévio para participação na pesquisa. Cada cão foi clinicamente avaliado, incluindo realização de exame neurológico, segundo Marcondes (2008) e otoscópico, conforme Cole (2004). Os donos foram questionados sobre histórico de uso de agentes ototóxicos e avaliação subjetiva da audição. Animais com alterações neurológicas, presença de ácaros ou cerúmen em excesso no conduto auditivo externo, ou com qualquer outra anormalidade, foram excluídos do estudo.

Para controle da variável temperatura, só foram incluídos no experimento animais com temperatura corporal entre $38,2^{\circ} \mathrm{C}$ e $39,2^{\circ} \mathrm{C}$. Para estudar eventuais alterações decorrentes do tamanho da cabeça, foram realizadas mensurações do diâmetro e comprimento dessa estrutura.

Potenciais evocados foram obtidos no Laboratório de Eletroneurodiagnóstico do Departamento de Clínica Veterinária, FMVZUnesp-Botucatu. Os animais foram mantidos em posição quadrupedal ou decúbito esternal, sem o uso de sedativos. Os eletrodos de registro foram colocados na região rostral ao tragus da orelha a ser testada (G1) e o de referência (G2) foi posicionado no vértice do crânio $(\mathrm{Cz})$ (Kay et al., 1984). O eletrodo terra foi colocado na região cervical dorsal (Eger e Lindsay, 1997). Foram utilizados filtros com banda passante de $200 \mathrm{~Hz}$ a $3.000 \mathrm{~Hz}$, sensibilidade de $2,5 \mu \mathrm{V} / \mathrm{cm}$ e varredura de $1 \mathrm{~ms} / \mathrm{cm}$. Fones de ouvido externos foram posicionados sobre as orelhas dos animais. Utilizaram-se "cliques" de rarefação de $0,2 \mathrm{~ms}$ de duração, intensidade de $85 \mathrm{~dB}$, apresentados na frequência de $13 \mathrm{~Hz}$, com mascaramento de ruído 
branco contralateral de 40dB. Em cada lado, foram obtidas pelo menos duas séries de 500 promediações utilizando-se equipamento Viasys Healthcare $^{\circledR}$, modelo Teca Synergy de dois canais.

Em cada teste foram medidas as latências absolutas em milissegundos das ondas I, III e V, bem como os intervalos I-III, III-V e I-V para cada lado. Foram calculadas as médias e o desvio-padrão das latências das ondas registradas nos exames para comparação dos valores entre os dois grupos. As análises estatísticas foram realizadas com o teste Mann Whitney $(\mathrm{P} \leq 0,05)$.

\section{RESULTADOS}

As médias das idades foram 3,6 (desvio-padrão: 1,78) e 10 anos (desvio-padrão: 1,68) para os animais dos grupos 1 e 2, respectivamente. As médias dos diâmetros das cabeças foram $9,38 \mathrm{~cm}$ (desvio-padrão: 1,09) para o grupo 1 e $9,58 \mathrm{~cm}$ (desvio-padrão: 1,46) para o grupo 2, respectivamente. A média dos valores de comprimento das cabeças foram $8,59 \mathrm{~cm}$ (desviopadrão: 1,35) e 8,64cm (desvio-padrão: 1,22) para os grupos 1 e 2 , respectivamente. Não houve diferença estatisticamente significativa para os valores de diâmetro $(\mathrm{P}=0,3144)$ e comprimento de cabeça $(\mathrm{P}=0,4573)$ entre os dois grupos.

A Fig. 1 ilustra os potenciais obtidos de um cão sem raça definida. Os valores referentes à média e ao desvio-padrão das latências absolutas em milissegundos das ondas I, III e $\mathrm{V}$ e os intervalos I-III, III-V e I-V para os diferentes grupos estão representados na Tab. 1. Não houve diferença estatística quando foram comparadas as latências médias das ondas I e III entre os dois grupos. As latências da onda $\mathrm{V}$ e dos intervalos III-V e I-V foram significativamente maiores no grupo de animais com idade acima de oito anos.

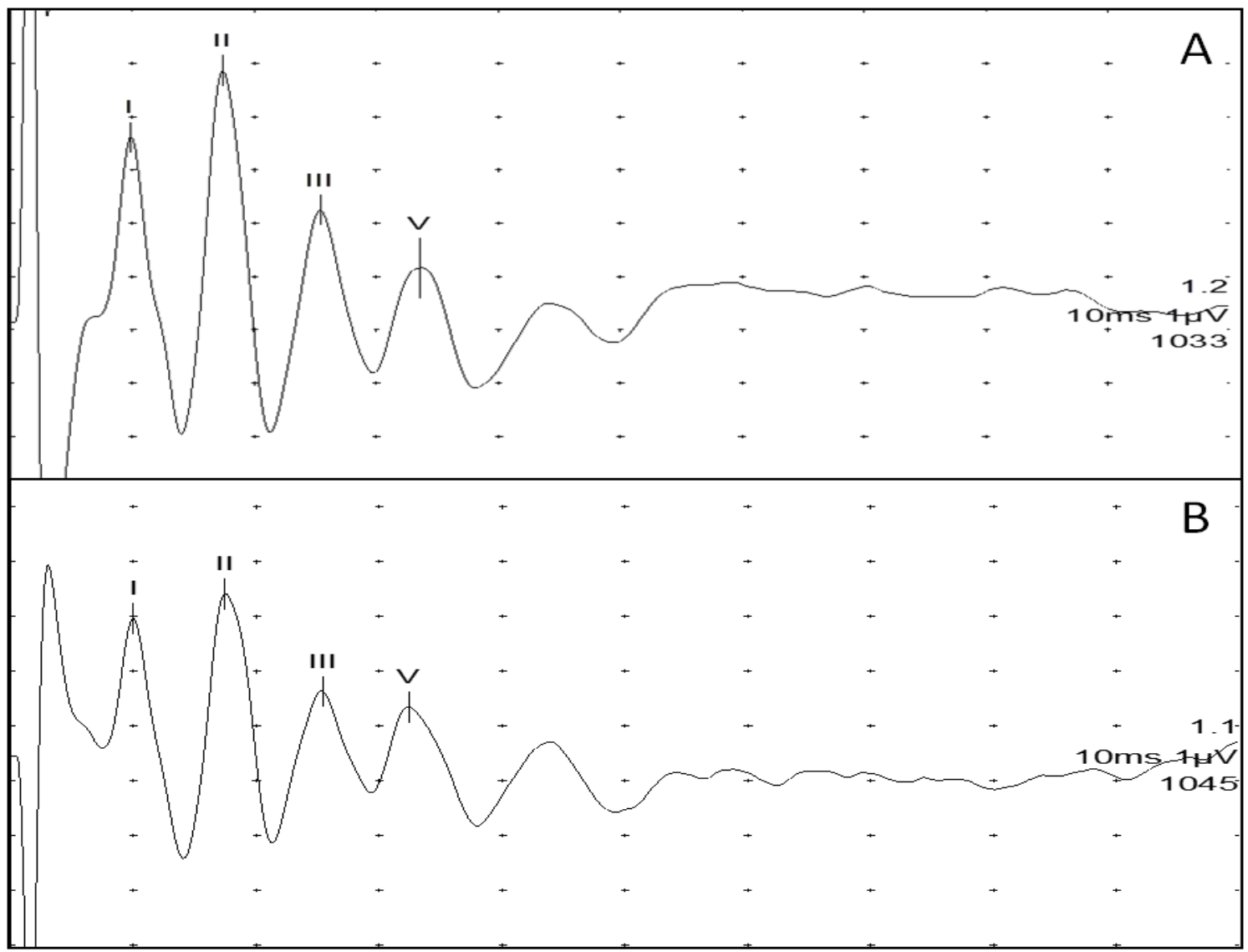

Figura 1. Potenciais evocados auditivos obtidos de um cão sem raça definida. A) Lado esquerdo; B) lado direito. Notar a marcação das ondas I, II, III e V (divisão vertical: $1 \mu \mathrm{V}$; divisão horizontal: $1 \mathrm{~ms}$ ). 
Tabela 1. Média e desvio-padrão das latências absolutas das ondas I, III e V e dos intervalos I-III, III-V e I-V, em milissegundos, dos diferentes grupos de cães sem raça definida

\begin{tabular}{ccccc}
\hline \multirow{2}{*}{ Latência } & \multicolumn{3}{c}{ GRUPO I } & \multicolumn{2}{c}{ GRUPO II } \\
\cline { 2 - 5 } & Média $(\mathrm{ms})$ & Desvio-padrão & Média $(\mathrm{ms})$ & Desvio-padrão \\
\hline I & $1,13 \mathrm{a}$ & 0,07 & $1,15 \mathrm{a}$ & 0,1 \\
III & $2,64 \mathrm{a}$ & 0,14 & $2,62 \mathrm{a}$ & 0,13 \\
V & $3,45 \mathrm{a}$ & 0,17 & $3,55 \mathrm{~b}$ & 0,15 \\
I-III & $1,51 \mathrm{a}$ & 0,14 & $1,47 \mathrm{a}$ & 0,15 \\
III-V & $0,81 \mathrm{a}$ & 0,11 & $0,93 \mathrm{~b}$ & 0,15 \\
I-V & $2,32 \mathrm{a}$ & 0,17 & $2,40 \mathrm{~b}$ & 0,11 \\
\hline
\end{tabular}

Médias seguidas de letras diferentes indicam diferença estatística entre os grupos $(\mathrm{P} \leq 0,05)$.

\section{DISCUSSÃO}

Filhotes de cães e gatos não nascem com o sistema auditivo completamente desenvolvido. As ondas I e II são as primeiras a aparecer, aproximadamente ao final da segunda semana de vida, e os valores de latências e amplitudes semelhantes a adultos são obtidos com seis a oito semanas de idade (Strain et al., 1991; Poncelet et al., 2000; Poncelet et al., 2002). Para que as alterações relacionadas com o desenvolvimento das vias auditivas não exercessem influência nos resultados deste trabalho, foram selecionados apenas animais com idades acima de um ano.

Muitos estudos relataram que o tamanho da cabeça influencia a amplitude e a latência das ondas (Allison et al., 1983; Trune et al., 1988; Pook e Steiss, 1990; Meij et al., 1992). No presente trabalho, não houve diferença estatística entre as características diâmetro e comprimento de cabeça entre os dois grupos; sendo assim, pode-se concluir que, apesar de se tratar de cães sem raça definida e com diferentes conformações de cabeça, essa característica não interferiu nos resultados obtidos.

Os valores das latências das ondas e dos intervalos observados neste estudo foram semelhantes a outros já definidos em cães por outros autores (Marshall, 1985; Myers et al., 1985; Knowles et al., 1988; Haagen et al., 1989; Harcourt-Brown et al., 2010), porém a intensidade de estímulo utilizada é diferente em cada trabalho. Sabe-se que as amplitudes e as latências das ondas variam de acordo com o nível de intensidade do estímulo auditivo (Strain et al., 1991; Luttgen, 1994), de maneira que intensidades crescentes de estímulo produzem queda correspondente na latência e aumento na amplitude das ondas observadas (Marshall, 1985; Strain et al., 1991; Luttgen, 1994; Eger e Lindsay, 1997; Cox, 2002). O presente estudo normativo utilizou estímulo de $85 \mathrm{~dB}$, e os resultados obtidos podem ser usados como valores de referência para a interpretação de exames realizados com essa mesma metodologia.

Alterações nos potenciais auditivos já foram relacionadas com a idade avançada em cães, como aumento da latência e redução da amplitude (Knowles et al., 1988), não identificação de ondas no registro (Knowles et al., 1989) e diminuição ou ausência de resposta à intensidade de 90dB (Shimada et al., 1998). No presente estudo, ao se compararem as latências dos dois grupos de animais, observou-se que apenas a onda $\mathrm{V}$ e os intervalos III-V e I-V foram maiores no grupo de animais mais velhos. Apesar da diferença de latência observada entre os dois grupos, não se encontrou dificuldade na marcação e na interpretação dos potenciais em nenhum exame, pois os resultados registraram ondas de fácil identificação.

Segundo Haar et al. (2008), a perda da audição relacionada com a idade em cães acontece por volta dos oito a 10 anos de idade e pode ser evidenciada com um aumento significativo no limiar auditivo. Os aumentos das latências observados nos animais com mais de oito anos do presente estudo pode ter ocorrido devido a alterações senis, porém o pequeno número de animais estudados e a não avaliação do limiar auditivo não permitem essa conclusão. Como a perda auditiva relacionada com a idade pode ser parcial, sua determinação requer a realização de exames audiométricos em diferentes frequências e intensidades de estímulos (Haar et al., 2008). 


\section{CONCLUSÕES}

Os registros realizados neste estudo não indicam que os animais mais velhos apresentavam deficiência auditiva, porém sugere-se que a idade deve ser levada em consideração na interpretação do BAEP, pois observou-se que as latências da onda $\mathrm{V}$ e dos intervalos III-V e I-V são mais tardias em animais acima de oito anos.

\section{AGRADECIMENTO}

Os autores agradecem o apoio fornecido pela Fapesp na forma de bolsa de estudo (Processo 2009/12290-8) e auxílio (Processo 2010/00518$1)$.

\section{REFERÊNCIAS}

ALLISON, T.; WOOD, C.C.; GOFF, W.R. Brain stem auditory, pattern-reversal visual, and short- latency somatosensory evoked potentials: latencies in relation to age, sex, and brain and body size. Electroenceph. Clin. Neurophy., v.55, p.619-636, 1983.

COLE, L.K. Otoscopic evaluation of the ear canal. Vet. Clin. North Small Anim. Pract., v.34, p.397-410, 2004.

COX, C. Investigation of hearing loss in dogs. In Practice., v.24, p.494-501, 2002.

EGER, C.E.; LINDSAY, P. Effects of otitis on hearing in dogs characterized by brainstem auditory evoked response testing. J. Small Anim. Pract., v.38, p.380-386, 1997.

HAAGEN， V.A.J.; SIEMELINK， R.J.G.; SMOORENBURG, G.F. Auditory brainstem response in the normal beagle. Vet. Q., v.11, p.129-137, 1989.

HAAR, G.T.; VENKER-VAN HAAGEN, A.J.; VAN DEN BROM, W.E. et al. Effects of aging on brainstem to toneburst auditory stimuli: a cross-sectional and longitudinal study in dogs. J. Vet. Intern. Med., v.22, p.937-945, 2008.

HARCOURT-BROWN, T.R.; PARKER, J.E.; GRANGER, N. et al. Effect of middle ear effusion on the brain- stem auditory evoked response of Cavalier King Charles Spaniels. Vet. J., v.188, p.341-345, 2010.
KAY, R.; PALMER, A.C.; TAYLOR, A.C. Hearing in the $\operatorname{dog}$ as assessed by auditoryevoked potentials. Vet. Rec., v.114, p.81-84, 1984.

KNOWLES, K.E.; CASH, W.C.; BLAUCH, B.S. Auditory-evoked responses of dogs with different hearing abilities. Can. J. Vet. Res., v.52, p.394-397, 1988.

KNOWLES, K.; BLAUCH, B.; LEIPOLD, H. et al. Reduction of spiral ganglion neurons in the aging canine with hearing loss. Zentralbl. Veterinarmed., v.36, p.188-199, 1989.

LUTTGEN, P.J. Deafness in the dog and cat. Vet. Clin. North Am. Small Anim. Pract., v.24, p.981-989, 1994.

MARCONDES, M. Semiologia do sistema nervoso de pequenos animais. In: FEITOSA, F.L.F. (Ed). Semiologia veterinária: a arte do diagnóstico. São Paulo: Roca, 2008. p.411-460.

MARSHALL, A.E. Brain stem auditory-evoked response of the nonanesthetized dog. Am. J. Vet. Res., v.46, p.966-973, 1985.

MARSHALL, A.E. Use of brain stem auditoryevoked response to evaluate deafness in a Dalmatian dogs. J. Am. Vet. Med. Assoc., v.188, p.718-722, 1986.

MEIJ, B.P.; VENKER-VAN HAAGEN, A.J.; VAN DEN BROM, W.E. Relationship between latency of brainstem auditory-evoked potentials and head size in dogs. Vet. Q., v.14, p.121-126, 1992.

MYERS, L.J.; REDDING, R.W.; WILSON, S. Reference values of the brainstem auditory evoked response of methoxyflurane anesthetized and unanesthetizes dogs. Vet. Res. Comm., v.9, p.289-294, 1985.

PONCELET, L.; COPPENS, A.; DELTENRE, P. Brainstem auditory evoked potential wave $\mathrm{V}$ latency-intensity function in normal Dalmatian and beagle puppies. J. Vet. Intern. Med., v.14, p.424-428, 2000.

PONCELET, L.C.; COPPENS, A.G.; DELTENRE, P.F. Audiograms estimated from brainstem toneevoked potentials in dogs from 10 days to 1.5 months of age. J. Vet. Intern. Med., v.16, p.674679, 2002. 
POOK H.A.; STEISS, J.E. Correlation of brain stem auditory-evoked responses with cranium size and body weight of dogs. Am. J. Vet. Res., v.51, p.1779-1783, 1990.

SHIMADA, A.; EBISU, M.; MORITA, T. et al. Age-related changes in the cochlear nuclei of dogs. J. Vet. Med. Sci., v.60, p.41-48, 1998.

SHIU, J.N.; MUNRO, K.J.; COX, C.L. Normative auditory brainstem response data for hearing threshold and neuro-otological diagnosis in the dog. J. Small Anim. Pract., v.38, p.103107,1997

STEISS, J.E.; COX, N.R.; HATHCOCK, J.T. Brain Stem Auditory-evoked response abnormalities in 14 dogs with confirmed central nervous system lesions. J. Vet. Int. Med., v.8, p.293-298, 1994.

STRAIN, G.M. Aetiology, prevalence and diagnosis of deafness in dogs and cats. Br. Vet. J., v.152, p.17-36, 1996.
STRAIN, G.M.; KEARNEY, M.T.; GIGNAC, I.J. et al. Brainstem auditory-evoked potential assessment of congenital deafness in Dalmatians: associations with phenotypic markers. J. Vet. Internal Med., v.6, p.175-182, 1991.

STRAIN, G.M.; TEDFORD, B.L.; JACKSON, R.M. Postnatal development of the brain stem auditory-evoked potential in dogs. Am. J. Vet. Res., v.52, p.410-415, 1991.

TRUNE, D.R.; MITCHELL, C.; PHILLIPS, D.S. The relative importance of head size, gender and age on the auditory brainstem response. Hearing Research., v.32, p.165-74, 1988.

UZUKA, T.; FURUTA, T.; YAMAOKA, M. et al. Threshold changes in auditory brainstem response $(A B R)$ due to the administration of kanamycin in dogs. Exp. Anim., v.45, p.325-331, 1996.

WEBB, A.A. Brainstem auditory evoked response (BAER) testing in animals. Can. Vet. J., v.50, p.313-318, 2009.

WILSON, W.J.; MILLS, P.C. Brainstem auditory-evoked response in dogs. Am. J. Vet. Res., v.66, p.2177-2187. 2005. 